\title{
Hard diffraction and parton rescattering
}

\author{
Rikard Enberg, Gunnar Ingelman, Roman Pasechnik \\ Department of Physics and Astronomy, Uppsala University \\ Box 516, SE-751 20 Uppsala, Sweden
}

DOI: http://dx.doi.org/10.5689/UA-PROC-2010-09/32

\begin{abstract}
We present a new model where hard diffraction is an effect of soft gluon rescattering between partons emerging from the hard interaction and the color field of the proton. A resummation to all orders of multiple gluon exchanges leads to an overall color singlet exchange amplitude which reproduces the precise HERA data on diffractive deep inelastic scattering.
\end{abstract}

\section{Introduction}

Hard diffraction is an active field of research, but the dynamics of the process is not completely understood from QCD. In hard diffractive scattering events, a beam proton escapes intact from the hard interaction with a large fraction of the beam momentum, even though a parton from the proton took part in a hard scattering subprocess with large momentum transfer. This requires the underlying process to involve an overall color singlet exchange. Experimentally, such events can be observed either through the leading proton that goes down the beam pipe and can be detected in very forward proton detectors placed along the beam, or through the presence of a large rapidity gap.

Hard diffraction was predicted in hadron-hadron collisions and interpreted in the pomeron language [1] in terms of hard scattering on a hadronic pomeron. The first observation of hard diffraction was by the UA8 experiment in $p \bar{p}$ collisions [2], as predicted in [1]. Much attention has been focused on diffractive deep inelastic scattering, which was discovered by the ZEUS and $\mathrm{H} 1$ experiments at HERA [3]. Diffractive DIS can be described in the pomeron model by parametrizing the parton content of the pomeron. However, the extracted parton densities are not universal, and when applied to diffractive hard scattering in $p \bar{p}$ collisions at the Tevatron one obtains cross sections an order of magnitude larger than observed.

This state of affairs led to the development of the Soft Color Interaction (SCI) model [4], based on the assumption that the hard perturbative part of a diffractive event is the same as in ordinary DIS. The central idea is then that the hard subprocess occurs on a scale that is much smaller than the bound state proton. The partons produced in the hard subprocess thus have to propagate through the color field of the proton and may interact with it. These long distance interactions do not affect the short distance interaction, but the exchange of color may change the color structure of the final parton state such that the hadronization leads to a different result. In particular rapidity gaps may be formed. The SCI model based on this principle is very successful in describing data [5], but lacks a solid theoretical basis.

For this reason, we have developed a new QCD-based model [6, 7], which leads to diffractive 


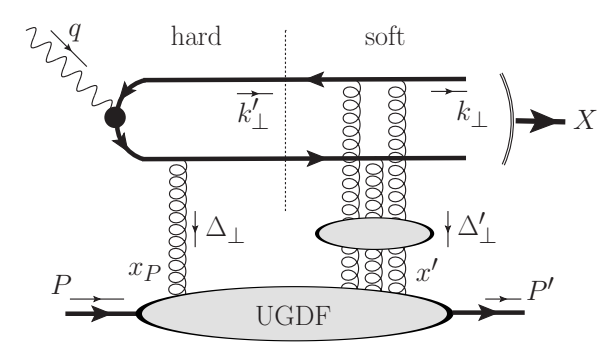

Figure 1: $\gamma^{*} p \rightarrow X p$ process with resummed gluon exchanges, and illustration of the factorization in Eq. (1) of the amplitude into a hard and a soft part connected via an unintegrated gluon density function (UGDF).

hard scattering through an effective overall color singlet exchange carried by multiple gluon exchanges. The model is inspired by the success of the SCI model, and by an earlier attempt [8] to understand this soft gluon exchange in terms of QCD rescattering.

\section{The model}

The main idea of the model is illustrated in Fig. 1, which shows how the exchanged virtual photon fluctuates into a quark-antiquark color dipole which interacts with the proton. The "hard" part of the process is defined by the interaction of the "first" gluon with longitudinal momentum fraction $x_{P}$. After this exchange, the $q \bar{q}$ dipole and the proton remnant have overall color octet charges. The hard part is described by conventional perturbative QCD.

The "soft" part of the process then consists of the interaction of the dipole with the remnant through the exchange of a number of soft gluons with longitudinal momentum fractions $x_{i}^{\prime} \ll$ $x_{P}, \sum x_{i}^{\prime}=x^{\prime}$. The soft part of the process includes at least one additional gluon exchange so that the overall exchange from both the hard and soft parts contains two gluons or more. These gluon exchanges are resummed to all orders, which leads to an exponential factor in the amplitude. The soft part is nonperturbative in nature and requires a treatment of soft, nonperturbative gluons.

The totality of the soft exchanges may form an overall color singlet system together with the first, hard gluon, in which case the proton remnant will be a color singlet that can recombine into a leading proton with close to the full beam momentum. The space-like photon has through the momentum exchange $x_{P}$ been turned into a time-like color singlet hadronic system $X$ of invariant mass $M_{X}$, and these two color singlet systems will be separated by a rapidity gap.

The full amplitude for $\gamma^{*} p \rightarrow X p$, including the hard and soft parts, can be written as a convolution in impact parameter space of the hard and soft subprocess amplitudes and a function $\mathcal{V}$, which describes the distribution of gluons in the proton,

$$
M(\delta) \sim \int d^{2} b e^{-i \boldsymbol{\delta} \cdot \mathbf{b}} \hat{M}^{\text {hard }} \hat{M}^{\text {soft }} \mathcal{V},
$$

where $\mathbf{b}$ is conjugate to $\boldsymbol{\Delta}_{\perp}$ and $\delta \equiv \sqrt{-t}=\left|\boldsymbol{\Delta}_{\perp}+\boldsymbol{\Delta}_{\perp}^{\prime}\right|$. The function $\mathcal{V}$ is the Fourier transform of the generalized unintegrated gluon distribution function (UGDF). This factorization is schematically illustrated in Fig. 1. 
The hard amplitude $\hat{M}^{\text {hard }}$ is computed in a standard way in perturbative QCD for transverse and longitudinal photon polarizations, and Fourier transformed into impact parameter space. The soft amplitude requires some more explanation (see $[6,7]$ for details). It can be calculated order-by-order and then resummed. However, the soft gluons carry nonperturbatively small momentum transfer, and there are thus three unknown aspects: the coupling to the quarks in the top part of the diagram, the coupling in the lower part of the diagram, which corresponds to a nonperturbative gluon density, and the propagator. For the nonperturbative propagator, we assume it is the same as the perturbative one. For the coupling to the quarks, we take $\alpha_{s}(\mu)$ at very small scales as a parameter, which we fix using analytic perturbation theory (APT) [9] at the scale $\Lambda_{\mathrm{QCD}}$, yielding $\alpha_{s}^{\text {soft }} \simeq 0.7$. Finally, to describe the coupling of the gluons to the proton, we use the framework of $k_{\perp}$-factorization and generalized UGDFs, which contain all information about the nonperturbative coupling of the gluons to the proton.

The needed generalized parton distributions are not very constrained by data. We therefore use a prescription [10] for the generalized UGDF, where the dependence on the longitudinal momentum fraction and transverse momentum of the soft gluons is incorporated in an explicitly symmetric way,

$$
\mathcal{F}_{g}^{\mathrm{off}} \simeq \sqrt{\mathcal{F}_{g}\left(x_{P}, \Delta_{\perp}^{2}, \mu_{F}^{2}\right) \mathcal{F}_{g}\left(x^{\prime}, \Delta_{\perp}^{\prime}{ }^{2}, \mu_{\mathrm{soft}}^{2}\right)}
$$

which explicitly involves the soft $x^{\prime}$ dependence. The first PDF $\mathcal{F}_{g}$ is the diagonal unintegrated gluon density, which depends on the gluon virtuality, and which is associated with the hard gluon. When integrated over the virtuality, it reduces to the conventional gluon PDF $g\left(x, \mu^{2}\right)$. As the dependence of $\mathcal{F}_{g}$ on the virtuality is not theoretically well-known for the small virtualities we encounter here, we model this UGDF by using the collinear gluon PDF together with a simple Gaussian Ansatz $f_{G}\left(\Delta_{\perp}^{2}\right)$ for the intrinsic transverse momentum dependence.

The second PDF in Eq. (2), associated with the soft gluons, is evaluated at very low scale and very small $x^{\prime}$. For this PDF we can introduce a function $\bar{R}_{g}\left(x^{\prime}, \mu_{\text {soft }}^{2}\right)$ which is assumed to be slowly dependent on $x^{\prime}$ in the case $x^{\prime} \ll x_{P}$ :

$$
\sqrt{x_{P}} \mathcal{F}_{g}^{\text {off }} \simeq \bar{R}_{g}\left(x^{\prime}, \mu_{\text {soft }}^{2}\right) \sqrt{x_{P} g\left(x_{P}, \mu_{F}^{2}\right)} f_{G}\left(\Delta_{\perp}^{2}\right) .
$$

The factor $\bar{R}_{g}$, therefore, contains all the soft physics related with soft gluon couplings to the proton. It is interpreted as the square root of the gluon PDF at very small $x^{\prime} \ll x_{P}$ and some soft scale $\mu_{\text {soft }}^{2}$. This is a nonperturbative object, which contributes to the overall normalization and can be determined from data. As we will see below, it is effectively a constant for most of phase space, and the prescription (3) is consistent with the HERA data for all available $M_{X}^{2}$ and $Q^{2}$.

The model (3) will lead to a linear dependence of the diffractive structure function on the gluon PDF, as compared to the quadratic dependence often encountered in two-gluon exchange calculations of DDIS [11]. This linear dependence is the same as in the SCI model, where a linear dependence describes both diffractive and non-diffractive events, and indicates a continuous transition between the two types of events. In terms of the UGDF in Eq. (3), the factor $\mathcal{V}=\mathcal{V}(\mathbf{b}, \mathbf{r})$ of Eq. (1) is given by

$$
\mathcal{V}(\mathbf{b}, \mathbf{r})=\frac{1}{\alpha_{s}\left(\mu_{\mathrm{soft}}^{2}\right)} \int \frac{d^{2} \Delta_{\perp}}{(2 \pi)^{2}} \sqrt{x_{P}} \mathcal{F}_{g}^{\mathrm{off}}\left\{e^{-i \mathbf{r} \boldsymbol{\Delta}_{\perp}}-e^{i \mathbf{r} \boldsymbol{\Delta}_{\perp}}\right\} e^{i \mathbf{b} \boldsymbol{\Delta}_{\perp}}
$$

We may now put all the pieces together and calculate [7] the final expressions for the 
diffractive structure functions in our model,

$$
\begin{aligned}
& x_{P} F_{L}^{D(4)}=\mathcal{S} Q^{4} M_{X}^{2} \int_{z_{\text {min }}}^{\frac{1}{2}} d z(1-2 z) z^{2}(1-z)^{2}\left|J_{L}\right|^{2} \\
& x_{P} F_{T}^{D(4)}=2 \mathcal{S} Q^{4} \int_{z_{\text {min }}}^{\frac{1}{2}} d z(1-2 z)\left\{(1-z)^{2}+z^{2}\right\}\left|J_{T}\right|^{2}
\end{aligned}
$$

where $\mathcal{S}=\sum_{q} e_{q}^{2} /\left(2 \pi^{2} N_{c}^{3}\right)$ sums over light quark charges $e_{q}$, and

$$
\begin{aligned}
& J_{L}=i \alpha_{s}\left(\mu_{F}^{2}\right) \int d^{2} \mathbf{r} d^{2} \mathbf{b} e^{-i \boldsymbol{\delta} \mathbf{b}} e^{-i \mathbf{r} \mathbf{k}_{\perp}} K_{0}(\varepsilon r) \mathcal{V}(\mathbf{b}, \mathbf{r})\left[1-e^{\mathcal{A W}}\right], \\
& J_{T}=i \alpha_{s}\left(\mu_{F}^{2}\right) \int d^{2} \mathbf{r} d^{2} \mathbf{b} e^{-i \boldsymbol{b} \mathbf{b}} e^{-i \mathbf{r k}_{\perp}} \varepsilon K_{1}(\varepsilon r) \frac{r_{x} \pm i r_{y}}{r} \mathcal{V}(\mathbf{b}, \mathbf{r})\left[1-e^{\mathcal{A W}}\right] .
\end{aligned}
$$

As we discuss in Ref. [7], we also include the emission of one extra gluon from the hard gluon propagator. This contribution is important at large $M_{X}$ only, and is dominated by collinear gluon exchange. We therefore model it using the DGLAP splitting function $P_{g g}$ as

$$
x_{P} F_{q \bar{q} g}^{D(4)} \simeq \frac{1}{N_{c}^{2}} \int \frac{d t_{g} d z_{g}}{t_{g}+m_{g}^{2}} P_{g g}\left(z_{g}\right) \frac{\alpha_{s}\left(t_{g}\right)}{2 \pi} x_{P} F_{q \bar{q}}^{D(4)},
$$

where $t_{g}$ is the gluon propagator and the integral is cut-off in the infrared by the effective gluon mass $m_{g} \simeq \Lambda_{\mathrm{QCD}}$. The factor $N_{c}^{-2}$ appears because the emitted gluon must contribute to the color singlet $X$ system.

\section{$3 \quad$ Numerical results}

The HERA data on the diffractive structure function [12] are given in terms of the reduced cross section,

$$
x_{P} \sigma_{r}^{D(3)}=x_{P} F_{q \bar{q}, T}^{D(3)}+\frac{2-2 y}{2-2 y+y^{2}} x_{P} F_{q \bar{q}, L}^{D(3)}+x_{P} F_{q \bar{q} g}^{D(3)}
$$

where $F_{L, T}^{D,(3)}\left(x_{P}, Q^{2}, \beta\right)$ is the diffractive structure function integrated over $t$, the kinematical variable $y=Q^{2} / s x_{B} \leq 1$, and the center-of-mass energy of ep-collisions at HERA is $\sqrt{s}=$ $318 \mathrm{GeV}$. In Fig. 2 we show the comparison of the results of our model with the latest HERA data [12] on the reduced cross section $x_{P} \sigma_{r}^{D(3)}\left(x_{P}, \beta, Q^{2}\right)$ as a function of $x_{P}$ in bins of $\beta$ and $Q^{2}$. The figure shows our main result, calculated using the CTEQ6L1 gluon PDF [13], and also curves obtained using the older GRV94 PDF [14]. The agreement with data is in general very good, except for very small $Q^{2}$ and $M_{X}$ where the QCD factorization scale is small and the PDFs are poorly known.

We find that transverse polarization dominates in all bins, and the $q \bar{q}$ contribution is enough to describe all the data for $\beta=Q^{2} /\left(Q^{2}+M_{X}^{2}\right) \gtrsim 0.2$, below which the $q \bar{q} g$ contribution becomes significant.

The parameters of our model are an effective gluon mass, $m_{g} \simeq \Lambda_{\mathrm{QCD}}$, which regulates the infrared divergence in the $q \bar{q} g$ contribution, Eq. (9), and the soft coupling constant $\alpha_{s}\left(\mu_{\text {soft }}^{2}\right) \simeq$ 0.7. There are also two physical quantities that we fit: the soft factor $\bar{R}_{g}$, and a constituent quark mass $m_{q}$. $\bar{R}_{g}$ is close to unity for a wide range of scales and thus $\bar{R}_{g}$ is essentially an 


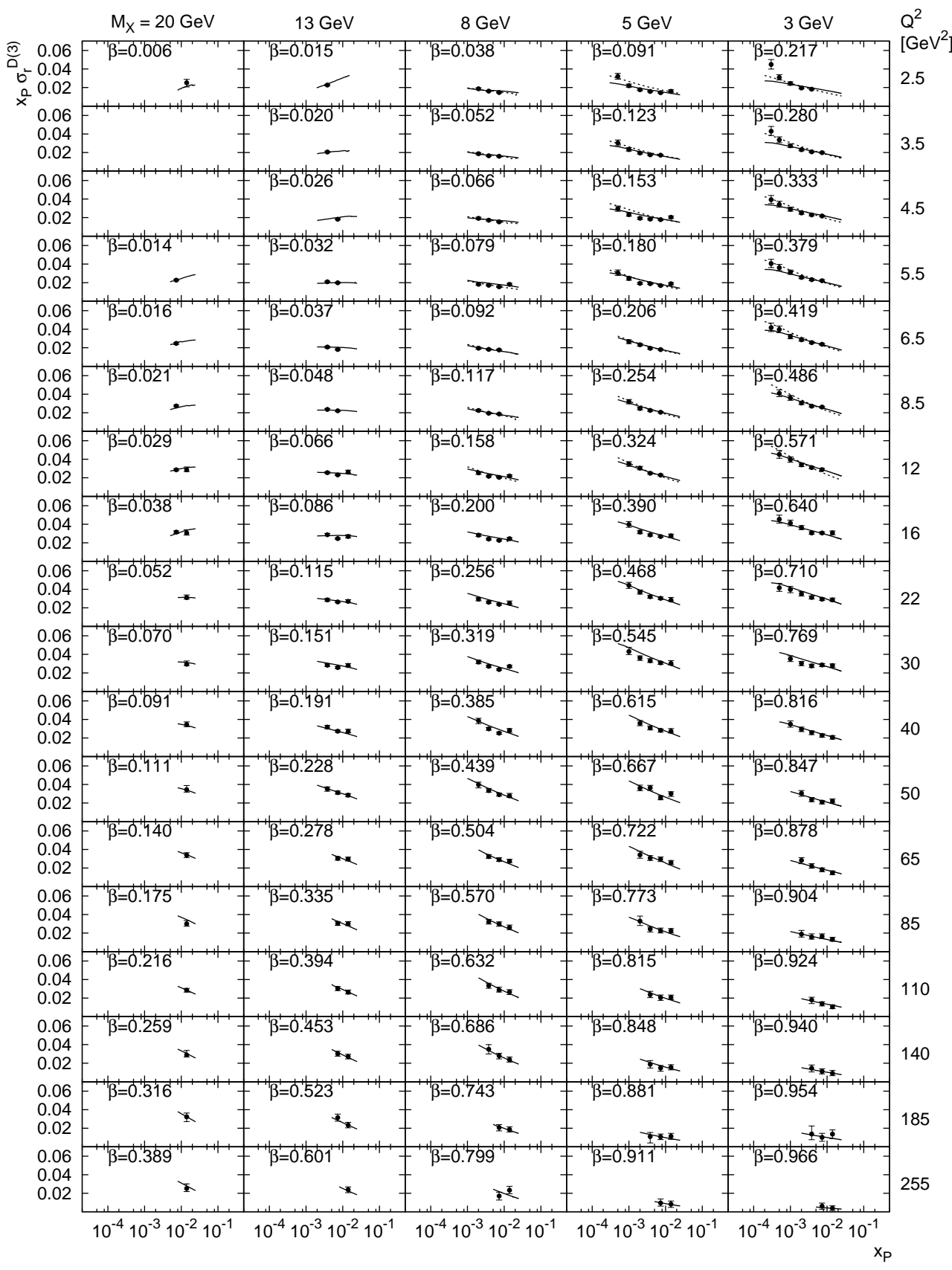

Figure 2: The reduced cross section $x_{P} \sigma_{r}^{D(3)}\left(x_{P}, \beta, Q^{2}\right)$ as a function of $x_{P}$ for different values of $M_{X}$ and $Q^{2}$. The latest ZEUS data [12], from diffractive deep inelastic scattering events with a large rapidity gap, compared with our model using the CTEQ6L1 (full line) and GRV94 (dotted line) parametrizations of the gluon density in the proton. 
overall normalization factor close to unity, which contains unknown information on the density of soft gluons in the proton. Again, we refer to [7] for a detailed discussion of these two quantities.

\section{Conclusions}

We have constructed a new QCD-based model for diffractive deep inelastic scattering, based on soft gluon exchanges in the final state, inspired by the phenomenologically successful Soft Color Interaction model [4] and the work on such soft rescattering in DIS in Refs. [15, 8]. The model describes data on the diffractive structure function very well. The model and the calculations are presented in full detail in $[6,7]$.

The gluon exchanges we consider arise from the color field of the proton and should therefore be more universal. We have studied diffractive DIS as a first step, but the model should also be applied to other processes, e.g. diffraction in hadron-hadron collisions or diffractive vector meson production. It should also be of importance for other observables, such as the underlying event at LHC. This is hinted at by the results obtained from the SCI model [5], which has not only been used to describe all diffractive data from HERA and the Tevatron, but also other, non-diffractive data.

\section{Acknowledgements}

This research was supported by the Swedish Research Council under contracts 2007-4071 and 2008-4137, and by the Carl Trygger Foundation.

\section{References}

[1] G. Ingelman and P. E. Schlein, Phys. Lett. B 152, 256 (1985).

[2] R. Bonino et al., Phys. Lett. B 211, 239 (1988).

[3] M. Derrick et al., Phys. Lett. B 315, 481 (1993); Phys. Lett. B 346, 399 (1995); T. Ahmed et al., Nucl. Phys. B 429, 477 (1994); Nucl. Phys. B 435, 3 (1995).

[4] A. Edin, G. Ingelman and J. Rathsman, Phys. Lett. B 366, 371 (1996); Z. Phys. C 75, 57 (1997).

[5] A. Edin, G. Ingelman and J. Rathsman, arXiv:hep-ph/9912539, in proc. 'Monte Carlo generators for HERA physics', DESY-PROC-1999-02 p. 280; R. Enberg, G. Ingelman and N. Tîmneanu, Phys. Rev. D 64, 114015 (2001); A. Edin, G. Ingelman and J. Rathsman, Phys. Rev. D 56, 7317 (1997); D. Eriksson, G. Ingelman and J. Rathsman, Phys. Rev. D 79, 014011 (2009).

[6] R. Pasechnik, R. Enberg and G. Ingelman, arXiv:1004.2912 [hep-ph].

[7] R. Pasechnik, R. Enberg and G. Ingelman, Phys. Rev. D 82, 054036 (2010).

[8] S. J. Brodsky et al., Phys. Rev. D 71, 074020 (2005).

[9] D. V. Shirkov and I. L. Solovtsov, Phys. Rev. Lett. 79, 1209 (1997).

[10] R. S. Pasechnik, A. Szczurek and O. V. Teryaev, Phys. Rev. D 78, 014007 (2008).

[11] M. Wusthoff and A. D. Martin, J. Phys. G 25, R309 (1999).

[12] S. Chekanov et al., Nucl. Phys. B 816, 1 (2009).

[13] J. Pumplin et al., JHEP 0207, 012 (2002).

[14] M. Gluck, E. Reya and A. Vogt, Z. Phys. C 67, 433 (1995).

[15] S. J. Brodsky et al. Phys. Rev. D 65, 114025 (2002). 